\title{
Microwave Processing of Planetary Surfaces for the Extraction of Volatiles
}

\author{
Edwin C. Ethridge ${ }^{1}$ \\ NASA Marshall Space Flight,Huntsville, AL, 35812 \\ and \\ William Kaukler ${ }^{2}$ \\ University of Alabama Huntsville, Huntsville, AL, 35899
}

\begin{abstract}
In-Situ Resource Utilization will be necessary for sustained exploration of space. Volatiles are present in planetary soils, but water by far has the most potential for effective utilization. The presence of water at the lunar poles, Mars, and possibly on Phobos opens the possibility of producing LOX for propellant. Water is also a useful radiation shielding material, and valuable to replenish expendables (water and oxygen) required for habitation in space. Because of the strong function of water vapor pressure with temperature, heating soil effectively liberates water vapor by sublimation. Microwave energy will penetrate soil and heat from within much more efficiently than heating from the surface with radiant heat. This is especially true under vacuum conditions since the heat transfer rate is very low. The depth of microwave penetration is a strong function of the microwave frequency and to a lesser extent on soil dielectric properties. Methods for complex electric permittivity and magnetic permeability measurement are being developed and used for measurements of lunar soil simulants. A new method for delivery of microwaves deep into a planetary surface is being prototyped with laboratory experiments and modeled with COMSOL MultiPhysics. We are planning to set up a planetary testbed in a large vacuum chamber in the coming year. Recent results are discussed.
\end{abstract}

\section{Introduction}

Water is one of the most plentiful compounds in the Universe. It is speculated that much of Earths water came from comets early in its history. Mars has vast quantities of water not only at the poles but also at lower latitudes. Significant quantities of water are present on several moons of Jupiter (Europa, Ganymede, and Callisto), Saturn (Enceladus), and Neptune (Titon). The Martian moon Phobos has a low density. For that reason some have speculated that it may have water ice or hydrated minerals.

In 1905 a Scientific American article speculated that there was water at the poles of the moon ${ }^{1}$. But, when the Apollo missions returned lunar soil samples, "no water" was present. In 1994, the SDI-NASA Clementine spacecraft mapped the surface of the moon. The microwave radio signals from shadowed craters at the lunar south pole were consistent with the presence of water. In 1998 neutron spectrometer data from Prospector showed high hydrogen concentrations at the poles. On October 9, 2009 the Lunar Crater Observation and Sensing Satellite (LCROSS) crashed into a crater at the south pole. LCROSS observations proved that there are large quantities of water at the lunar poles.

The presence of water on planetary bodies is of high importance. Water and oxygen are required expendables for extended human exploration. Estimates from architectural studies of a lunar outpost indicated that one ton of water and one ton of oxygen would be needed each year of operation. The estimated cost for the delivery of payloads to the lunar surface is $\$ 100,000$ per pound. Use of in-situ water could greatly reduce costs for manned exploration. Another significant potential application for in-situ water is as a source for $\mathrm{H}$ and $\mathrm{O}$ for use as an in-space propellant. Water is also recognized for its radiation protection potential. It is only slightly less effective as a radiation shield than polyethylene. Lunar water is likely to be the first in-situ resource material that gains significant utilization for exploration activities. The dramatic impact by LCROSS on the water laden creater at the lunar south pole proved there is tremendous quantity of water frozen in the polar regolith. In a NASA Science News article of March 18, 2010, Paul Spudis remarked on the LCROSS findings:

"If you converted those craters' water into rocket fuel, you'd have enough fuel to launch the equivalent of one space shuttle per day for more than 2000 years.

\footnotetext{
${ }^{1}$ Materials Scientist, Materials \& Processes Lab., EM41, AIAA non-Member.

${ }^{2}$ Assistant Research Professor in Chemistry, AIAA non-Member.
} 


\section{Background}

For six years we have been conducting applied research associated with the use of microwaves for in-space applications. In particular we are developing the use of microwaves for the extraction of volatiles from planetary surfaces. Other volatiles may also be extracted by microwave processes. But, our discussions in the remainder of this paper will concentrate on water extraction since it has the most immediate potential for use in exploration projects and since it is known that water exists in large concentrations on many "planetary" surfaces.

Microwave processing has several advantages. Soil (in a vacuum or near vacuum) is an excellent thermal insulator. Astronauts measured the thermal conductivity of lunar regolith at two landing sites. The measured thermal conductivity ranges from 150 to $300 \mathrm{~W} / \mathrm{cm}-\mathrm{K}$. Reflecting sunlight onto the surface only heats a thin top layer of soil. Since heat flow into the regolith is very slow, this would not be an effective method for volatiles extraction.

Microwave energy will penetrate the soil heating from the inside out. The microwave penetration depth is dependent on frequency and on the dielectric properties of the soil. The absorbed microwave energy heats the soil which subsequently heats the water ice which sublimes to water vapor and percolates through the soil to be captured by a cold trap. Since the soil can be heated in situ, excavation may not be required. The generation of plumes of hazardous lunar dust could be minimized. Also at the cryogenic temperatures of the lunar poles, the frozen permafrost could be as hard as granite. Complex mechanical operations to dig the soil and processing chambers proposed for other extraction processes would not be required.

In our prior work, it was demonstrated that microwave energy will couple to cryogenically cooled lunar soil simulant (JSC-1A) containing 1\% water-ice. ${ }^{2}$ The permafrost simulant was contained in a fused silica vessel, our "moon in a bottle", Figure 1, for this proof of principle experiment. As the cold permafrost simulant was heated from LN2 temperatures, the water ice sublimed (less than 2 minutes). This water vapor left the soil and traveled along the line connected to a vacuum pumping station. An inline cold trap between the two captured virtually all of the liberated water. This proof of principle experiment validated the concept of microwave heating of lunar soil to extract water. It was demonstrated that microwaves $(2.45 \mathrm{GHz})$ will couple with lunar soil simulant (that does not contain nano-phase iron) at cryogenic temperatures. The resulting heating of the simulant caused the water ice to rapidly sublime and could be collected in a cold trap with high efficiency.

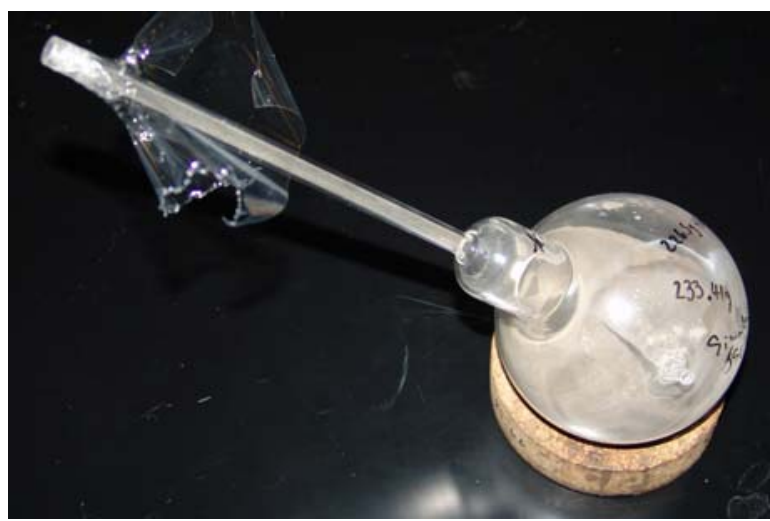

Figure 1. Our "Moon in a Bottle" fused silica (microwave transparent) test article used to hold the lunar regolith permafrost simulant while a vacuum is pulled on the glass tube. Liquid nitrogen was poured over the sample. The sample was placed in a domestic microwave oven to heat the permafrost.

Heating of the soil depends on absorption of the microwave energy. The extent of microwave absorption by soil is dependent on several factors. The penetration depth is greatly affected by the microwave frequency. Long wavelengths (lower frequencies) penetrate deeper than short wavelength (higher frequencies). Knowledge of the dielectric properties is required to calculate the penetration depth (see Table I) and to model soil heating and water extraction. The dielectric properties are also a function of temperature. But, in general, there is less absorption at colder temperatures. The dielectric properties are also dependent on microwave frequency. There is less absorption at low frequencies $(0.5 \mathrm{GHz})$ and much higher absorption and heating at high frequencies $(10 \mathrm{GHz})$. 
Table 1. Real and Imaginary Permittivity for five materials used in the water extraction experiment, illustrating the dependence of microwave $(2.45 \mathrm{GHz})$ penetration on the properties. Note that microwaves penetrate deeper into ice than simulant illustrating that microwaves are not absorbed by ice as much as by the regolith simulant.

\begin{tabular}{|l|l|l|l|}
\hline & \multicolumn{1}{|c|}{ e $^{\text {e }}$} & \multicolumn{1}{|c|}{} & Penetration \\
\hline Water & 0.1 & 8 & $6 \mathrm{~cm}$ \\
\hline Simulant JSC-1 & 0.1 & 4 & $10 \mathrm{~cm}$ \\
\hline Ice & 0.001 & 3 & $1 \mathrm{~m}$ \\
\hline Polyethylene & 0.0001 & 2 & $100 \mathrm{~m}$ \\
\hline quartz & 0.00001 & 3 & $1000 \mathrm{~m}$ \\
\hline
\end{tabular}

It has been reported, that Apollo lunar regolith couples very well with microwave energy ${ }^{4}$. Lunar soil contains very small nano-phase metallic iron (n-Fe) spheres in the surface layer of regolith particles. It is said that n-Fe causes significant coupling with microwaves which aids the heating of lunar soil. For this reason the Lunar Simulant Workshop ${ }^{5}$ identified the importance of replicating the microwave dielectric properties of lunar soil as with lunar simulants.

The 2004 Lunar Simulant Workshop ${ }^{5}$ in Huntsville concluded that a daughter simulant derived from JSC-1a should be developed to simulate the effect of nano-phase iron. Other investigations (Orbitec, Inc, Plasma Processes, etc.) have produced daughter simulants containing agglutinates and some n-Fe. By developing the methods for measuring electromagnetic properties, we can evaluate how these daughter simulants compare with JSC-1A and with Apollo lunar soil. An important goal is to determine if the n-Fe in lunar soil has a sufficient effect on the dielectric properties to justify production of n-Fe containing simulants.

\section{Dielectric Property Measurements}

The response to an electromagnetic field caused by the dielectric properties of a material (complex electric permittivity and to a lesser degree permeability), are critical to understanding the interaction of microwaves with the regolith and the design of microwave heating and processing systems. Comparison of microwave dielectric properties with actual lunar soil, ultimately will be needed to evaluate the adequacy of lunar simulants for prototype experiments. The frequency dependence of microwave penetration into lunar regolith is needed for the design of space experiments and optimal selection of processing conditions for volatiles extraction. Since we are modeling permafrost containing water ice, it should be emphasized that microwave coupling to ice is two orders of magnitude less than that of water. Therefore, one can neglect the effects of water ice on the dielectric properties.

Dielectric measurements of Lunar soil are reported in the Lunar Sourcebook ${ }^{3}$ for the low RF range $102-107$ $\mathrm{Hz}^{3,6,7}$ with some measurements at $9.3 \mathrm{GHz}^{8}$ and many above $10 \mathrm{GHz}^{3}$. Many of these measurements are from remote sensing. To our knowledge there are no laboratory measurements of the complex electric (dielectric) permittivity and complex magnetic permeability in the 1 to $10 \mathrm{GHz}$ range for Apollo lunar soil. Also, as far as we know, no magnetic permeability measurements of any kind have ever been made with lunar soil and regolith simulant materials.

The first method we developed for making these measurements is the transmission line filled waveguide method. Microwave waveguide fixtures had to be designed and fabricated from purchased parts, see Figure 2. The waveguides were connected to coaxial instrumentation signal cables to the network analyzer. An Agilent programmable network analyzer (PNA, model number E8361A with A.06.04.30 firmware) from the Microwavemillimeter Instrument Characterization Laboratory at the MSFC Materials \& Processes Laboratory was utilized for the measurement of complex electric permittivity and magnetic permeability. A dedicated software package (Agilent 85071 version E2.24.02 Materials Measurement Software from Innovative Solutions Inc.) takes the data from the analyzer and fits it to appropriate models to extract the desired properties. A considerable learning curve 
had to be overcome to setup the fixtures for the sensitive measurements without introducing artifact signals as well as to determine which of the embedded PNA software modules to use.

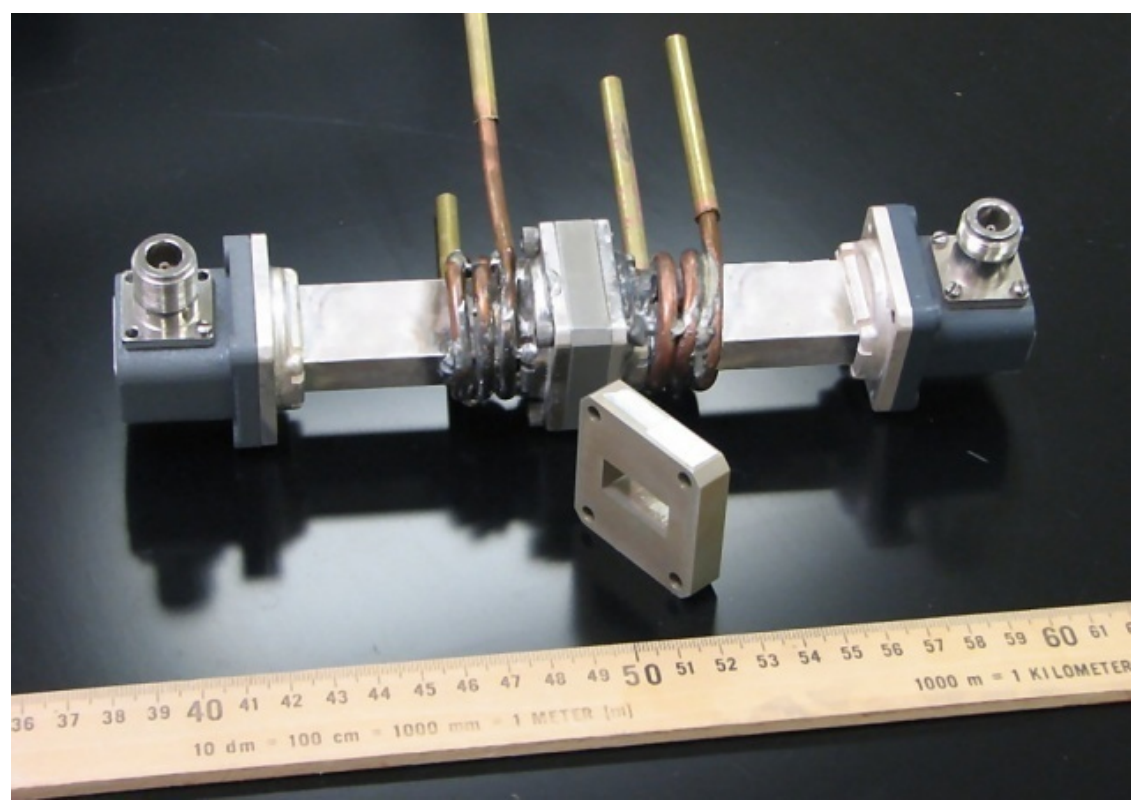

Figure 2. An X-band (10 GHz) test fixture for dielectric measurements using the transmission/reflection filled waveguide method. The sample is placed in a sample holder and mounted in the center of the fixture. Mica sheets are used to contain the simulant power.

A second measurement method, resonant cavity perturbation ${ }^{9,10}$, has been under development by Agilent. WK has been working with Agilent for its implementation in our laboratory. This resonant method is appropriate for measuring small samples. It is especially suitable for measurements of Apollo lunar soil samples, which we hope to have the opportunity to measure. Another advantage is the sensitivity of the method to the complex dielectric constant. This method is claimed to be more accurate than the transmission line technique for permittivity of lowloss materials such as lunar regolith simulant.

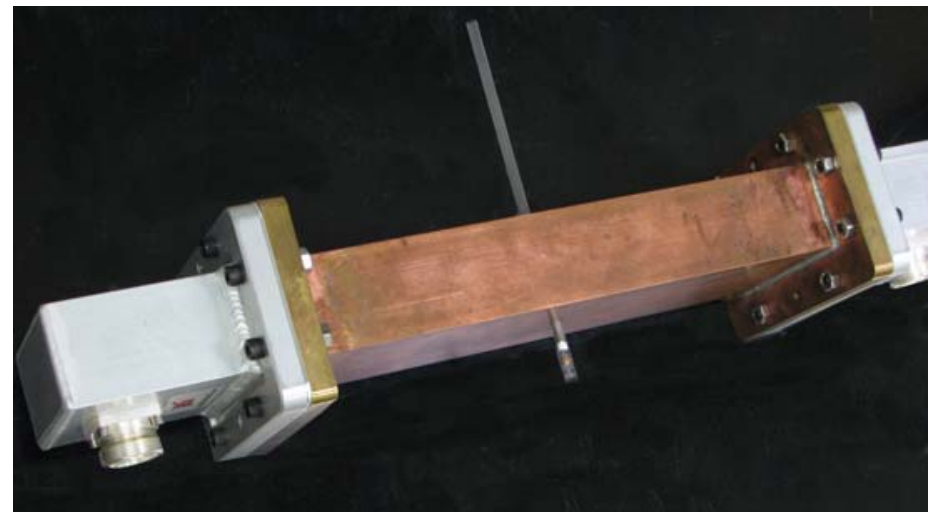

Figure 3. An S-band $(2.45 \mathrm{GHz})$ resonant cavity fitted with the coax to waveguide adapters at each end. Through the midpoint of the waveguide, a sample holding tube passes through the waveguide. Waveguide coupling apertures not visible, are sized empirically for these measurements. The copper waveguide is about one foot long.

Since complex permeability measurements in the microwave region are very difficult, significant time and effort has been required to refine these methods and to verify the performance and accuracy. Another problem is that accepted standard materials do not exist. This required identification of materials with known magnetic permeability that could serve as standards for our measurements. 


\section{PERMITTIVITY AND PERMEABILITY MEASUREMENTS}

We have measured the complex electric permittivity and magnetic permeability of several lunar soil simulants. The baseline standards are the lunar mare simulants JSC-1 and JSC-1A. A daughter simulant, JSC-1A AGGL, was produced by Orbitec, Inc. which contains agglutinates with nano-phase elemental iron in the thin surface layer. Transmission microscopy of the samples show spherical nano-phases that look very much like those in lunar soil. The USGS NU-LHT lunar highlands and Orbitec's JSC-Mars-1A simulant were also measured.

In order to evaluate the possible effects of elemental iron on the permittivity (and especially on permeability), we made samples of JSC-1A containing 1 weight $\%$ iron power in two different forms. The first (JSC-1A 1\%FE) is JSC-1A simulant with 200 micrometer sized metallic iron powder mechanically mixed together. A second (JSC-1A 1\%CIP) is JSC-1A mixed with Carbonyl Iron Powder (CIP) with 3 to 5 micrometer particles. Since the iron in these mixtures is not contained within the surface layers of the regolith particles, it is different from the nano-phase iron in lunar regolith.

Measurements were made of "standard" materials, regolith simulants, and other materials, but, for this paper we will discuss only simulants containing iron. Permittivity and permeability measurements at $2.45 \mathrm{and} 10 \mathrm{GHz}$ are shown in Table II for several regolith simulants, all measured at room temperature. Other measurements have been made at temperatures well below room temperature but will not be reported at this time. Methods for cryogenic temperature measurements are still being developed. Our goal is to obtain the properties of simulants at the microwave frequencies $12-8 \mathrm{GHz}, 2.2-3.3 \mathrm{GHz}$, and $0.9 \mathrm{GHz}$, at room temperature and at cryogenic temperatures.

Table II. Complex Electric Permittivity (e' + ie”) and Complex Magnetic Permeability (u’+ iu”) For Regolith Simulants and daughter simulants containing metallic iron.

\begin{tabular}{|c|c|c|c|c|c|c|c|c|}
\hline $2.45 \mathrm{GHz}$ & $\mathrm{e}^{\prime}$ & e" & $\mathrm{u}^{\prime}$ & u" & $10 \mathrm{GHz} \quad \mathrm{e}^{\prime}$ & $e^{\prime \prime}$ & $\mathrm{u}^{\prime}$ & u" \\
\hline JSC-1A & 3.9 to 4.2 & 0.08 & 1.007 & 0.001 & 3.7 & 0.08 & 1 & 0.001 \\
\hline JSC-1A 1\%CIP & 4.23 & 0.08 & 1.016 & 0.003 & 3.8 to 4.1 & 0.2 & 1.1 & 0 \\
\hline JSC-1A 1\%Fe & 4.00 & 0.00 & 1.00 & 0.00 & & & & \\
\hline JSC-1A AGGL & 3.8 & 0.059 & 1.011 & 0.001 & 3.2 to 3.7 & 0.5 & 1.05 & 0 \\
\hline NU LHT & 3.34 & 0.021 & 1.007 & 0.001 & 3.2 & 0.004 & 1 & 0 \\
\hline JSC-1 & 3.8 & 0.09 & 1.01 & 0.002 & & & & \\
\hline JSC-Mars-1A & 3.37 & 0.0252 & 1.008 & 0.002 & & & & \\
\hline Jensen & 4.28 & 0.168 & 1.011 & 0.001 & & & & \\
\hline
\end{tabular}

A few representative raw data plots for four of the simulants using the waveguide transmission method in the $\mathrm{X}$-band, are shown in Figures 4 to 7 below. One can see the difficulty in selecting a single value for each of the four constants. The measurements are sensitive to noise causing artifacts (peaks and dips in the data). Sometimes using a different software tool can minimize the artifacts. The property values, actually, do not change radically within the bandwidth of the measurements, so a flat region near 2.45 or $10 \mathrm{GHz}$ was selected for the property value. The values in Table II represent an average of values from the best measurements. What is clear from the data is that there is no significant effect of small concentrations of metallic iron on the measured permittivity and permeability in comparison with JSC-1A. 


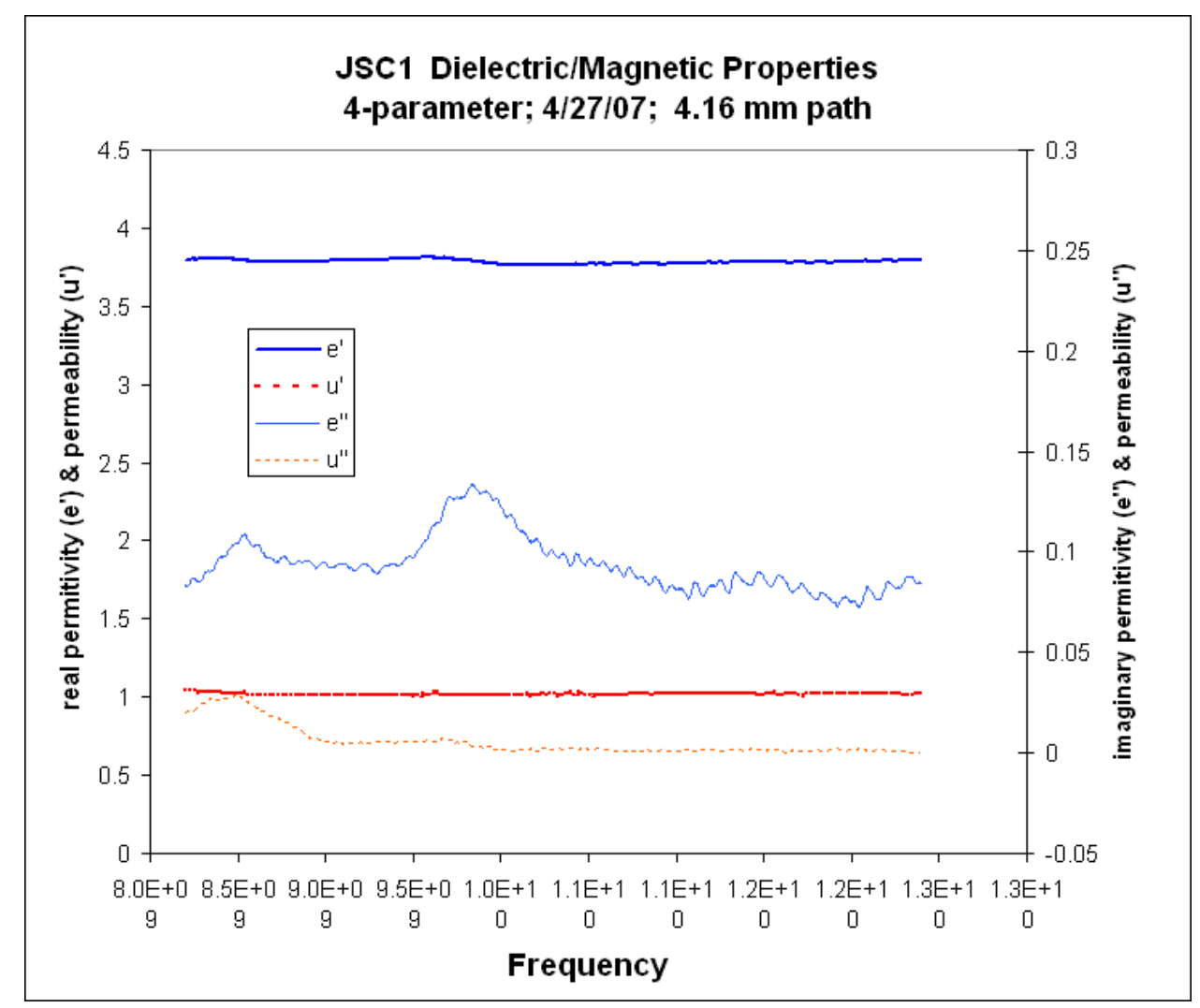

Figure 4. Complex electric (dielectric) permittivity (e' and e") and the complex magnetic permeability for JSC$1 \mathrm{~A}$ in the microwave $\mathrm{X}$-band $(10 \mathrm{GHz})$.

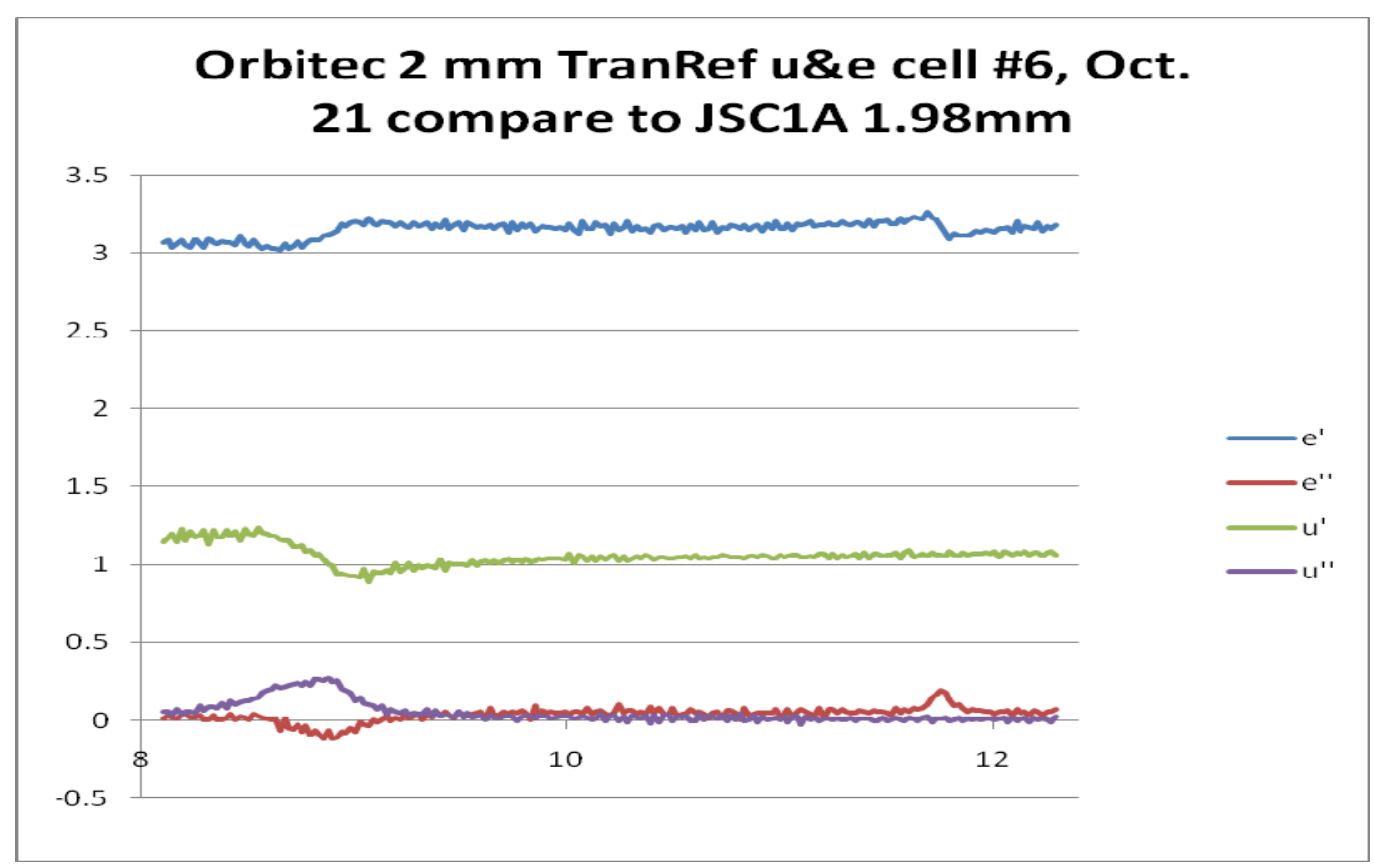

Fig. 5 Orbitec JSC-1A AGGL with aggultinates and n_Fe, 2 mm thick. X-band transmission line waveguide using transmission and reflection algorithm providing both real and complex permittivity e' and e" as well as permeability u' and u”. 


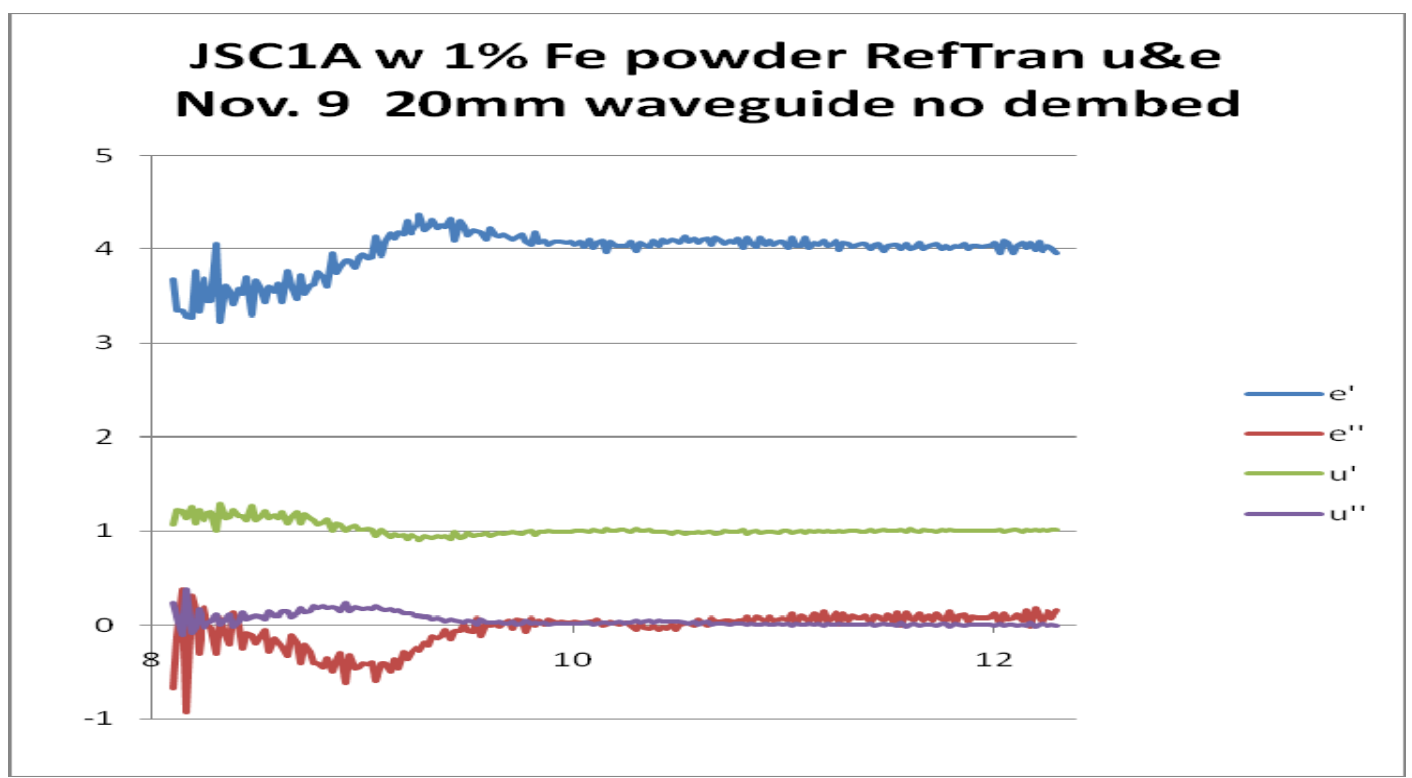

Fig. 6 JSC-1A plus $1 \mathrm{wt} \%$ iron powder (200 micron typical particle size) sample, $20 \mathrm{~mm}$ thick. X-band data using waveguide transmission and reflection algorithm that providing both real and complex permittivity and permeability.

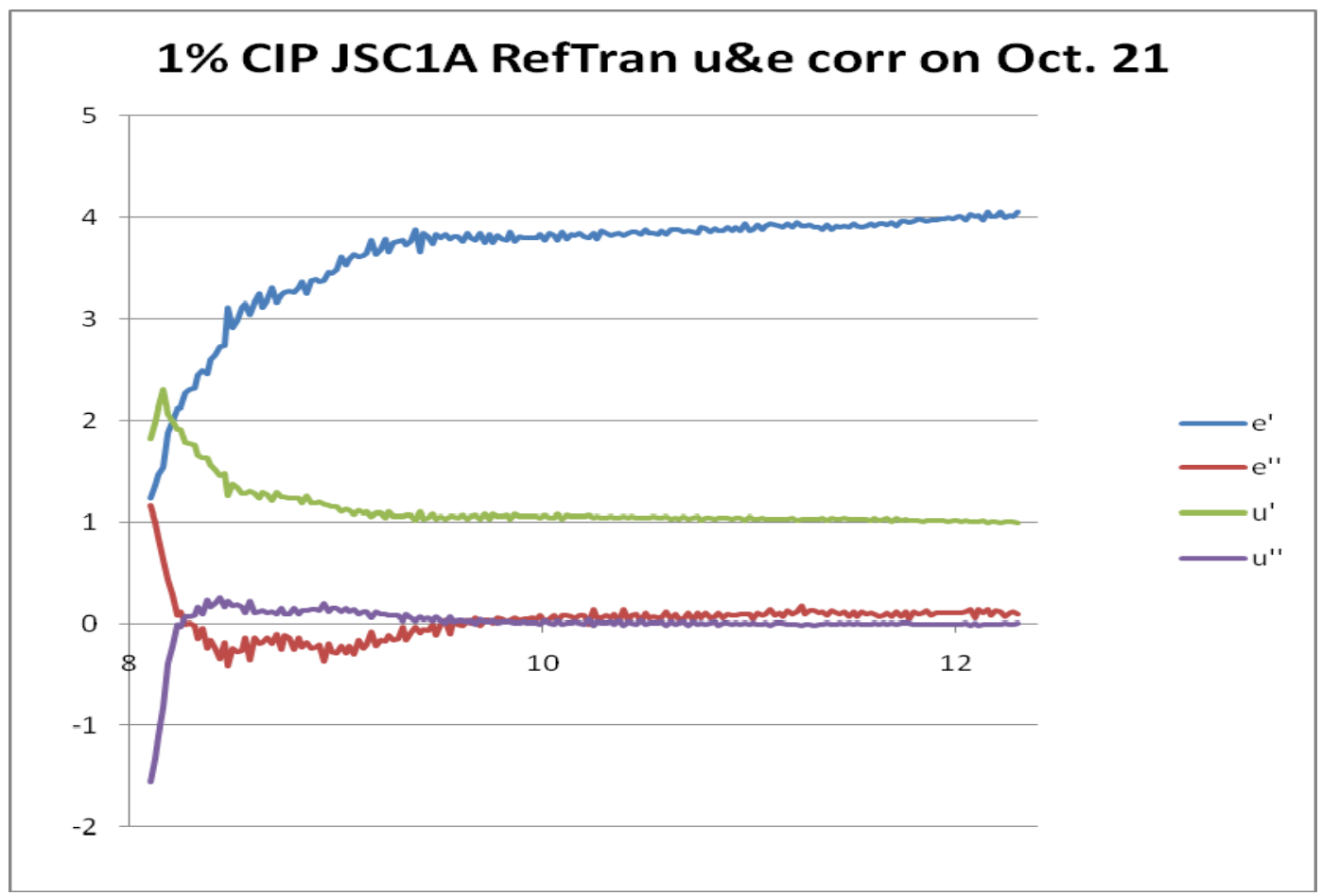

Figure 7. JSC-1A plus $1 \mathrm{wt} \%$ iron powder (200 micron typical particle size), $20 \mathrm{~mm}$ thick. X-band transmission line waveguide using transmission and reflection algorithm providing both real and complex permittivity and permeability. 


\section{COMSOL FEA Modeling}

In previous microwave heating experiments with lunar simulant JSC-1A, temperatures were measured at different depths in the simulant. It was shown that the microwave energy did attenuate into the soil according to Beer's Law. But, it became obvious that experimental setups for experiments at different microwave frequencies would be both time consuming and expensive. Interpretation of the measured results is also difficult.

One of us, EE, had used COMSOL to model an International Space Station experiment, so it was decided to perform finite element modeling of the microwave heating experiments. Our numerical modeling of soil heating with COMSOL Finite Element Analysis (FEA) Multiphysics software, utilizes two physics software modules. First, the RF Module is used to calculate the penetration of microwaves into a volume of soil. The microwave frequency is fixed for each calculation. Dielectric properties measured for JSC-1A lunar soil simulant are used for the calculations. The model design was tested with numerous trial calculations until parameters were determined that permitted the calculations to converge. The three-dimensional model illustrates how the microwave energy and power density decreases as it penetrates into the soil. The next step in the model development is the addition of the heat flow physics model. The thermal conductivity values measured for lunar soil from Apollo measurements and other soil properties were estimated using values for similar materials such as igneous rock. The calculated microwave energy absorbed by the soil simulant in the RF module is used by the Heat Transfer module to calculate soil heating and temperature isotherms. The resulting coupled multiphysics 3-D calculation demonstrates how the soil heats with time.

Preliminary results from these calculations have been very revealing. The frequency of the microwave energy has a strong effect on the energy absorbed by the soil and specifically on the depth and extent of heating of the soil. $^{11}$ Three dimensional plots of temperature isotherms from microwave heating at three different microwave frequencies are shown in Figure 8 for comparison. One can see the much greater penetration at $10 \mathrm{GHz}$ in comparison to 2.45 and $0.5 \mathrm{GHz}$. 


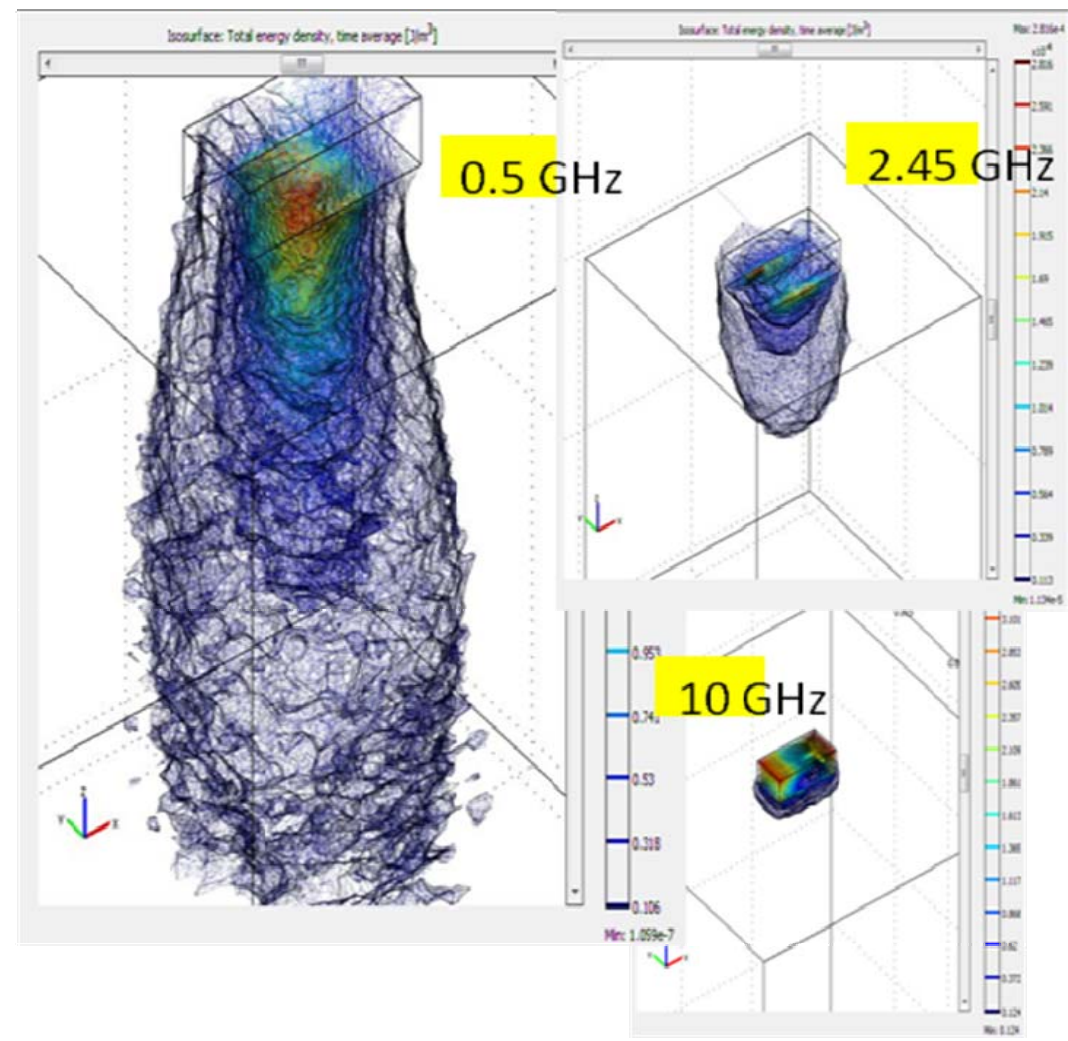

Figure 8. COMSOL MultiPhysics FEA calculations of microwave energy penetration into lunar regolith simulant at three different microwave frequencies, $0.5,2.45$, and $10 \mathrm{GHz}$.

The most important thing learned from the numerical modeling is that one can calculate the heating processes in the soil for different experimental and processing conditions. Multiphysics FEA modeling permits the calculation of heating effects depending on specific soil dielectric properties, thermal properties, and the temperature dependence of those properties. The modeling also permits one to calculate the heating effects based on the microwave frequencies, power level, and length of time of heating. It will be much less expensive to perform parametric calculations than to perform many different types of microwave hardware and setup laboratory experiments. Once general optimal experimental parameters are known, then more definitive experiments can be performed. This kind of understanding of the physics underlying the microwave heating of regolith to extract volatiles will be extremely important for experiment design and hardware requirements needed to design experiments both in the lab and on planetary surfaces.

\section{Development of Volatile Extraction Methods}

We have been developing water extraction methods both by experimentation and with numerical modeling. The first system was prototyped to get an understanding of how a microwave system might be constructed and to test system components. It directs microwaves $(2.45 \mathrm{GHz})$ down directly into the lunar regolith simulant, see Figure 9. A magnetron generates the microwaves that pass though a waveguide to a high gain horn antenna with good forward gain. A three stub tuner was used for impedance matching, but was not required since the microwave energy efficiently couples into the regolith. Heating within the simulant bed was measured with Luxtron fluorooptic thermometry. The temperature increase was consistent with simple calculations of attenuation.

In a parallel effort, COMSOL MultiPhysics was used to model the microwave penetration and heating into a regolith bed. Figure 9 shows a 3-D model for the process with temperature isotherms within the 3-D box below the microwave source. The calculation illustrates how useful numerical modeling of microwave systems can be to gain an understanding of microwave heating of regolith analogues. 

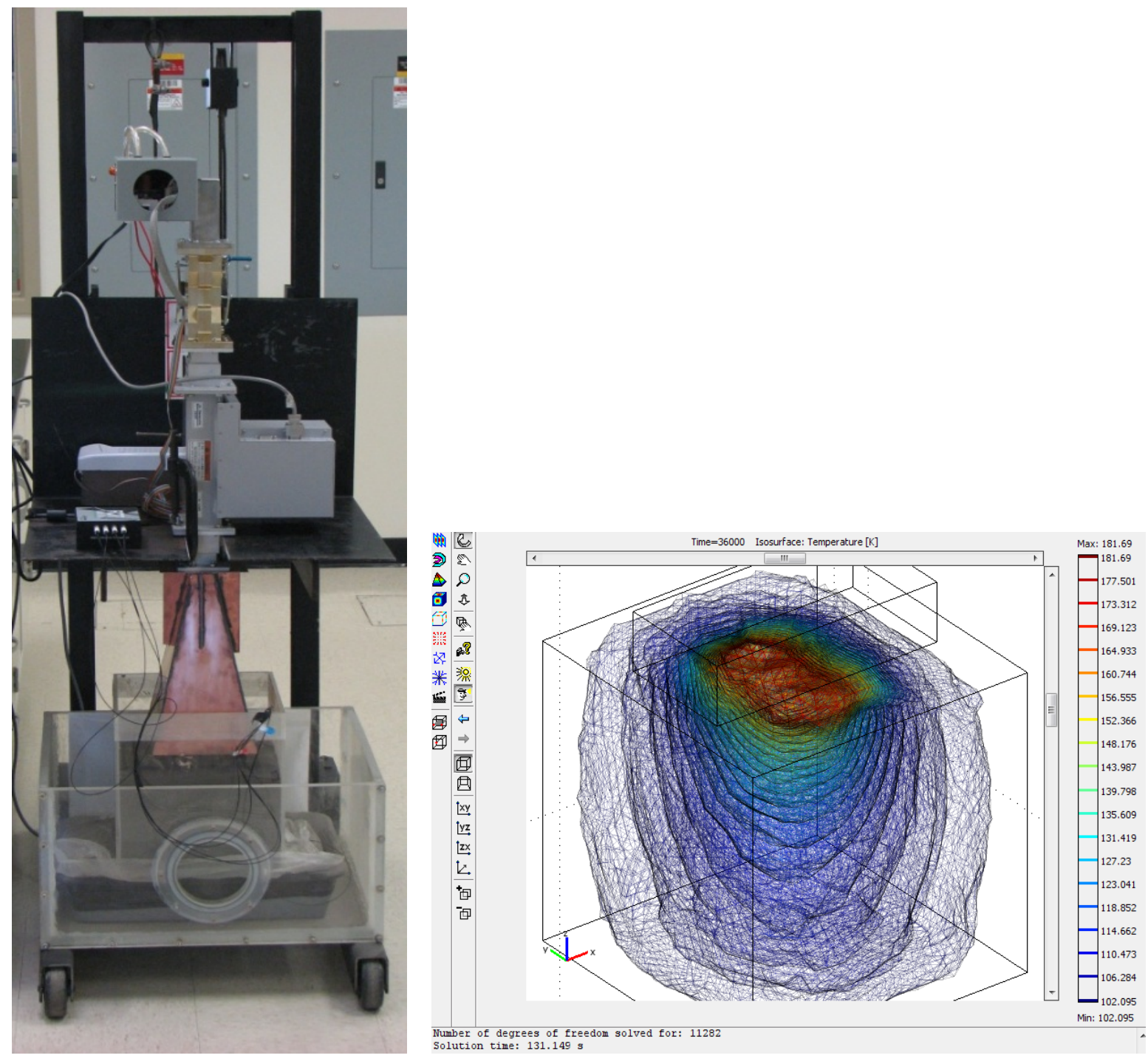

Figure 9. On the left is our laboratory setup for directing microwave energy into a planetary surface analogue. On the right is a COMSOL MultiPhysics calculation showing the penetration of microwave energy and heating isotherms (colored isobars).

In another laboratory experiment, we will evaluate a new method for getting microwaves deep below the surface, through a hypothetical compacted top layer that is depleted of water ice. A vacuum system is connected to a vacuum ring attached to a 1 meter chamber, see Figure 10. Microwave energy will be delivered down 1 meter thorough the regolith where the microwave energy will heat the surrounding regolith simulant containing ice and other volatiles. The water vapor and other volatile species that are liberated will be measured with a mass spectrometer. A schematic drawing in Figure 10 shows the inside of the vacuum system. 

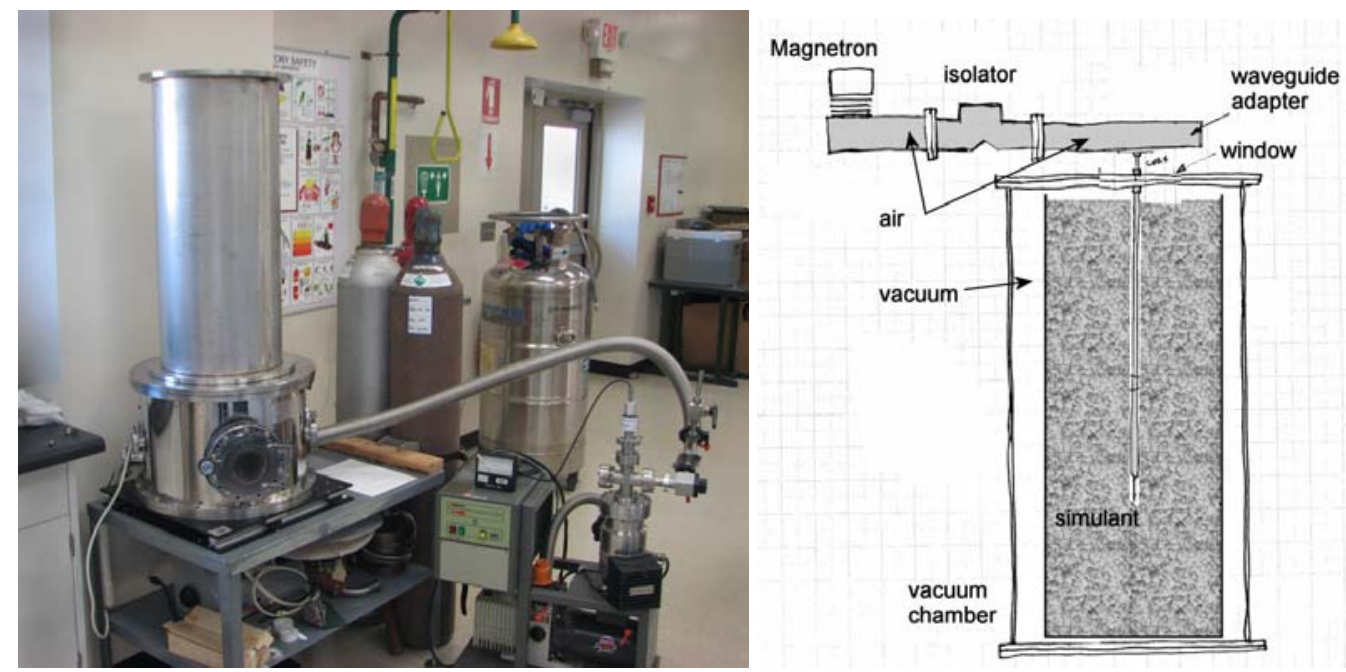

Figure 10. On the left is a laboratory setup of a vacuum system for testing of the extraction of water from 1 meter below the top surface of a bed of regolith simulant permafrost. On the right is a schematic drawing of the experimental setup for testing the permeability of lunar simulant and water extraction from 1 meter deep.

This extraction method is also being modeled with COMSOL. The axi-symmetric model has a microwave source 1 meter from the top surface. The microwave source 1 meter deep, which emits microwaves into the regolith, absorbed by the regolith, heating from the initial temperature of $173 \mathrm{~K}$. Figure 11 shows 3 frames from a time sequence. The color boundary between white and red is the $373 \mathrm{~K}$ isotherm where there should be no water remaining in the white region. The heated region out to beyond yellow is where water vapor would be rapidly subliming.
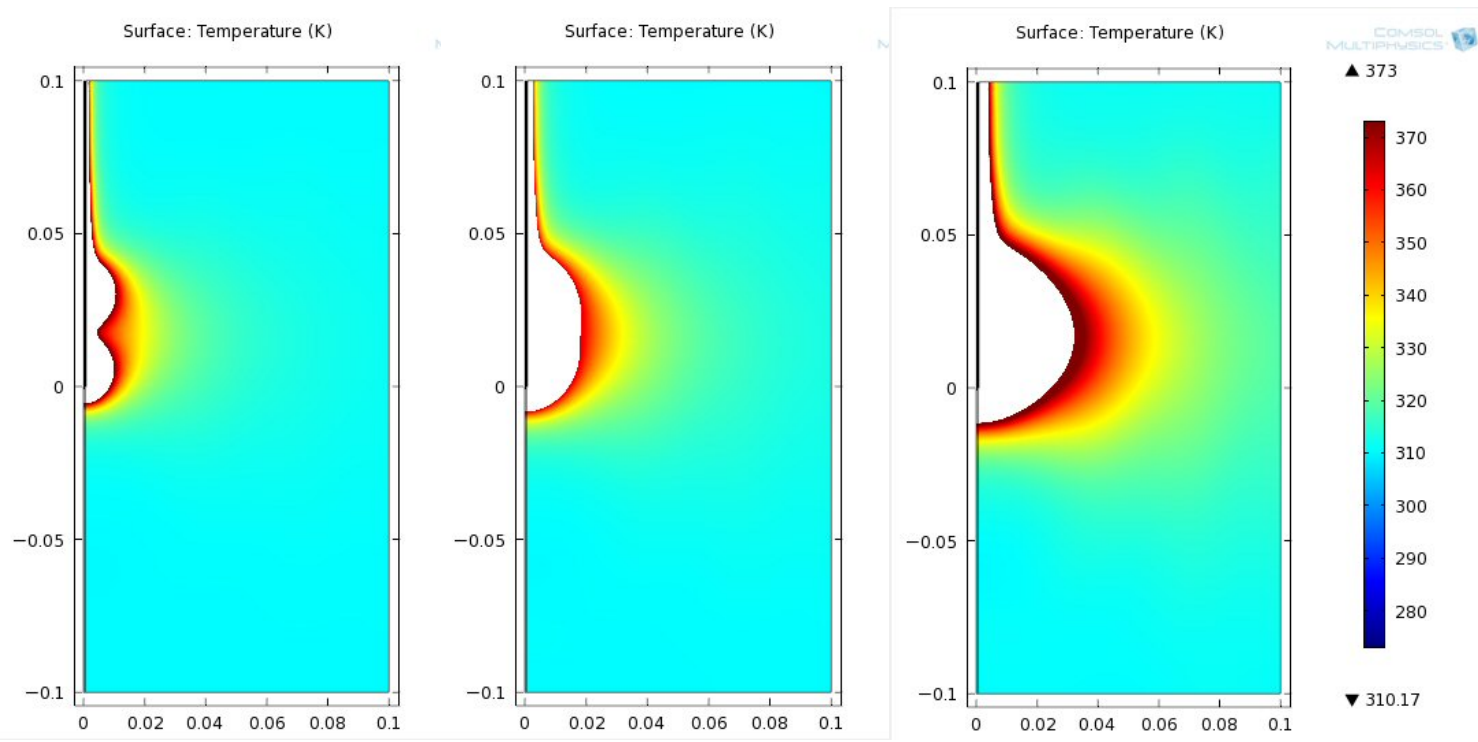

Figure 11. Transient COMSOL FEA axi-symmetric calculations of a time sequence with microwave heating at the bottom of a bore hole into a planetary body. The three frames from left to right are at 600, 1200, and 3600 seconds.

\section{Other Work in Progress}

The permeability of water vapor through regolith is an unknown that could greatly limit the microwave water extraction process. A study has begun in collaboration with University of Alabama Huntsville and Southern Research Institute (SRI) to evaluate gas flow through JSC-1A and NU-LHT regolith simulants at a range of densities 
(compactions) from 1.5 to $2.0 \mathrm{~g} / \mathrm{cm}^{3}$. SRI has performed permeability measurements according to the ASTM Standard 1434-82 ${ }^{12}$. The data is being evaluated to determine metrics for possible water vapor extraction rates. Representative raw data from this study is given in Figure 12, which shows the Darcy coefficient for JSC-1A over a range of pressure differences for a compacted regolith with a density of $1.73 \mathrm{~g} / \mathrm{cm} 3$.

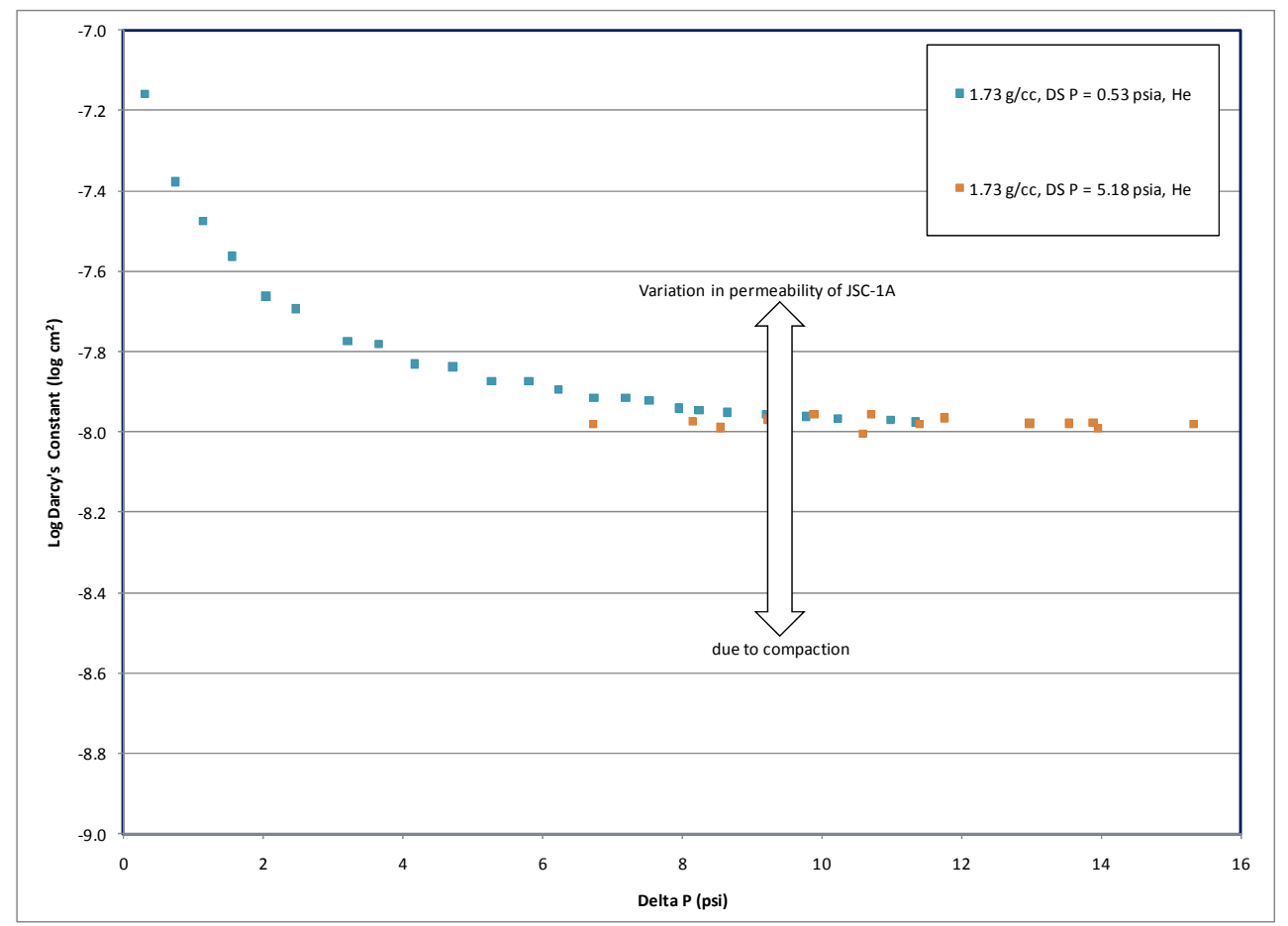

Figure 12. Darcy’s Constant for JSC-1A (1.73 g/cm3) over a range of pressure differences.

A COMSOL model is being developed for a 3-D calculation of the water vapor flow rate through regolith simulant. The COMSOL model will be validated with experimental data. Then, we anticipate coupling the physics of water vapor sublimation and the permeability data to the existing COMSOL Multiphysics model to build a universal numerical model for the microwave water extraction process.

\section{Conclusion}

1. Microwaves $(2.45 \mathrm{GHz})$ will couple with lunar regolith simulants at cryogenic temperatures, even without nano-phase metallic iron particles present in lunar soil.

2. Water ice sublimes well below 0C, and can be captured efficiently in an external cold trap.

3. The dielectric properties of lunar regolith simulants have been measured.

4. For the several regolith simulants containing metallic iron, there appears to be no significant difference in the four materials constants, real and complex electric permittivity and the real and complex magnetic permeability when compared with JSC-1A.

5. Prototype microwave systems have been assembled to test coupling of microwaves to lunar regolith to deliver microwave energy into lunar regolith simulant, and heating to extract volatiles.

6. Microwave energy penetration, absorption, and heating correlate with calculations.

7. COMSOL multiphysics is useful for modeling microwave penetration at different microwave frequencies and microwave heating with processing time.

8. COMSOL will be useful for parametric studies of different scenarios for microwave heating of different planetary surfaces. 


\section{Acknowledgments}

We wish to thank NASA Headquarters for the financial support of this Lunar Advanced Science and Exploration Research (LASER) research project. Special thanks to MSFC Management for prior seed funding that permitted us to develop the initial concepts. We also acknowledge Walter Frei at COMSOL for his technical assistance getting the COMSOL models setup.

\section{References}

${ }^{1}$ Anomous, “Is there life on the moon”, Scientific American Volume XCII, No 26, p 511, December 23, 1905.

${ }^{2}$ Ethridge, E. and W. Kaukler, "Extraction of Water from Extraterrestrial Bodies - Dielectric Property Measurements”, AIAA 47th Aerospace Sciences Meeting, Orlando, FL, January 5 to 82009

${ }^{3} \mathrm{~T}$ Lunar Sourcebook, Lunar and Planetary Institute, 1991

${ }^{4}$ Taylor, L.A. and Meek, T.T., "Microwave sintering of lunar soil: Properties, theory, and practice”, J. Aerospace Engr., 18(3), 188-196, 2005.

${ }^{5}$ Lunar Regolith Simulant Workshop, Huntsville, AL, 2004

${ }^{6}$ Strangway, D.W., Pearce, G.W., and Olhoeft, G.R., Magnetic and dielectric properties of lunar samples, in The SovietAmerican Conference on the Cosmochemistry of the Moon and Planets: National Aeronautic and Space Administration SP-370 p. 417-432., 1977

${ }^{7}$ Chung, D. H., Westphal, W. B., and Olhoeft, G. R.; Dielectric properties of Apollo 14 lunar samples; Proc. Third Lunar S Science Conference, supplement to Geochimica et Cosmochimica Acta, v3, pp3161-3172, MIT Press, 1972

${ }^{8}$ Bassett, H. L. and Shackelford, R. G.; Dielectric properties of Apollo 14 lunar samples at microwave and millimeter wavelengths; Proc. Third Lunar Science Conference, supplement to Geochimica et Cosmochimica Acta, v3, pp3157-3160, MIT Press, 1972

${ }^{9}$ Kumar, A., S. Sharma, and G. Singh, "Measurement of Dielectric Constant and Loss Factor of the Dielectric Materials at Microwave Frequencies,” Prog. Electromagnetics Res., PIER 69, 47-54, 2007

${ }^{10}$ American Standards for Testing Materials, ASTM A893/A893M-03 "Standard Test Method for Complex Dielectric Constant of Nometallic Magnetic Materials at Microwave Frequencies"

${ }^{11}$ Ethridge, E. and W. Kaukler, “Using Microwaves for Extracting Water from the Moon”, COMSOL Conference, Keynote presentation, Boston, MA October 9, 2009.

${ }^{12}$ ASTM D 1434-82, "Standard Test Method for Determining Gas Permeability Characteristics of Plastic Film and Sheeting” ASTM International, West Conshohocken, PA 2003. 